Abstract 01-S02.02 Table 2 Unadjusted and adjusted estimates of HPV detection on incidence of other STls (total number of visit pairs $=3221$ )

\begin{tabular}{|c|c|c|c|c|}
\hline \multicolumn{2}{|c|}{$\begin{array}{l}\text { Detected with the } \\
\text { following at previous } \\
\text { visit }\end{array}$} & $\begin{array}{l}\text { New cases of } \\
\text { other STIs, } n=46 \\
(1.4 \%) n(\text { row } \%)\end{array}$ & \multicolumn{2}{|c|}{$\begin{array}{l}\text { New detection of other } \\
\text { STIs across consecutive visits }\end{array}$} \\
\hline \multicolumn{5}{|c|}{ Any HPV } \\
\hline No & $2590(80.4)$ & $31(1.2)$ & 1.0 & 1.0 \\
\hline \multirow[t]{2}{*}{ Yes } & $631(19.6)$ & $15(2.4)$ & 1.94 (1.05 to 3.58$)$ & 1.81 (0.94 to 3.49$)$ \\
\hline & & $\begin{array}{l}\text { New cases } \\
\text { of other STIs, } \\
n=46(1.4 \%) \\
n \text { (row\%) }\end{array}$ & \multicolumn{2}{|c|}{$\begin{array}{l}\text { New detection of other STIs across } \\
\text { consecutive visits }\end{array}$} \\
\hline \multicolumn{5}{|c|}{ Any HR-HPV } \\
\hline No & $2930(91.0)$ & $38(1.3)$ & 1.0 & 1.0 \\
\hline Yes & $291(9.0)$ & $8(2.7)$ & $2.14(1.00$ to 4.61$)$ & $2.00(0.82$ to 4.83$)$ \\
\hline
\end{tabular}

Estimates adjusted for age and study site at enrolment, as well as the following covariates assessed at each follow-up visit: pap smear diagnosis at previous visit, contraceptive use in last 6 months, number of lifetime partners, partners having sex with others in last 6 months, having new partner in last 12 months, male partner using condom in last 6 months. Covariates that were found to be statistically significantly associated with the outcomes $(p<0.05)$ and/or significantly influence the effect size of the primary association of interest $(\geq 10 \%)$ were included in the final models for confounding control. Parity, smoking status, and other factors measured, such as age of sexual debut and frequency of sex in last 6 months, did not satisfy these criteria in the data analyses and hence werenot included in the final models.

HPV, human papillomavirus; HR-HPV, High-risk HPV, defined as HPV types 16, 18, 31, 33, $35,39,45,51,52,56,58,59,66$ (IARC 2007); STls, sexually transmitted infections.

Methods Women aged 20-38 years were followed semi-annually for 18 months in Thailand $(n=1200)$. Assessment was made on cervical HPV genotypes, cervical cytology, sexual behaviour, demographic factors and diagnoses of other STIs including chlamydia, gonorrhoea, syphilis, genital herpes and trichomoniasis. Incident detection was defined as any type-specific HPV or other STI which was detected at current visit but not at previous visit. Associations were measured by ORs with $95 \%$ CIs estimated in generalised estimating equation models.

Results During follow-up, 241 new cases of HPV, 110 incident cases of high risk (HR)-HPV and 46 new cases of other STIs were observed. Diagnosis of other STIs at previous visit was statistically significantly associated with twofold increased odds of any new HPV detection after controlling for sexual behaviour, age, pap smear status and contraceptive use [adjusted OR (aOR): any HPV: 2.16 (95\% CI: $1.08 \%$ to $4.34 \%$ )] (Abstract O1-S02.02 table 1 ). No significant association was found between diagnosis of other STIs and subsequent incident detection of HR-HPV [aOR: 2.01 (95\% CI: $0.74 \%$ to $5.48 \%$ )] (Abstract O1-S02.02 table 1). Positive detection of any HPV or HR-HPV predicted nearly twofold increased odds of other STIs with the estimates bordering on statistical significance [aORs: any HPV: 1.81 (95\% CI: $0.94 \%$ to $3.49 \%$ ); HR-HPV: 2.00 (95\% CI: $0.82 \%$ to $4.83 \%$ )] (Abstract O1-S02.02 table 2).

Conclusions We show that other STIs increase the risk of HPV incidence after controlling for sexual behaviour. The data qualitatively suggest mutual associations of HPV with other STIs. Further studies are warranted to evaluate if these reflect true biologic interactions between HPV and other sexually transmitted microbial agents, or mere confounding from unmeasured sexual risks.

\section{1-S02.03 MULTIPLE SEX PARTNERS AND LACK OF CONDOM USE FOR ANAL SEX ARE ASSOCIATED WITH MULTIPLE ANAL HPV INFECTIONS AMONG MEN HAVING SEX WITH MEN: THE HIM STUDY}

doi:10.1136/sextrans-2011-050109.9

${ }^{1}$ A Nyitray, ${ }^{2} \mathrm{~L} L$ Villa, ${ }^{3} \mathrm{R} J$ Carvalho da Silva, ${ }^{2} \mathrm{M}$ L Baggio, ${ }^{1} \mathrm{~B}$ Lu, ${ }^{1} \mathrm{D}$ Smith, ${ }^{1} \mathrm{M}$ Abrahamsen, ${ }^{1} \mathrm{M}$ Papenfuss, ${ }^{4} \mathrm{E}$ Lazcano-Ponce, ${ }^{1} \mathrm{~A}$ R Giuliano. ${ }^{1} \mathrm{H}$. Lee Moffitt
Cancer Center and Research Institute, Tampa, USA; ${ }^{2}$ Ludwig Institute for Cancer Research, São Paulo, Brazil; ${ }^{3}$ Centro de Referência e Treinamento em DST/AIDS, São Paulo, Brazil; ${ }^{4}$ Instituto Nacional de Salud Pública, Cuernavaca, Mexico

Background Human Papillomavirus (HPV) infection is the primary cause of anal cancer. While multiple HPV infections in the anal canal may accelerate disease progression, there are no reports of behavioural factors associated with multiple anal HPV infections among men having sex with men (MSM) and men having sex with women (MSW). We hypothesised that infection with multiple HPV types in the anal canal among MSM was associated with multiple sex partners and lack of condom use for recent anal sex. For comparison, we also assessed the role of multiple partners and condom use among MSW.

Methods Genotyping for $37 \mathrm{HPV}$ types was conducted on anal canal exfoliated cell specimens from men, ages 18-70, from São Paulo, Brazil; Cuernavaca, Mexico; and Tampa, Florida, USA. Specimens from the pre-enrolment visit of a 4-year prospective study were analysed. Eligibility included no history of genital warts and no current STD diagnosis including HIV. Exfoliated cell samples between the anal verge and the dentate line of the anal canal were obtained with a saline-wetted swab. A total of 193 MSM and 1407 MSW provided evaluable specimens. For multivariable analyses we used Poisson regression with a robust sandwich estimator. Association estimates were adjusted for potential confounders.

Results Multiple HPV infections were present in the anal canal of $34.7 \%$ of MSM and $4.0 \%$ of MSW. Prevalence of multiple HPV infections was stable by age group among MSW ( $p$ trend $=0.65$ ) but declined among MSM ( $p$ trend=0.009). After adjustment for potential confounders, $\geq 2$ male anal sex partners in the past 3 months (OR $2.47,95 \%$ CI $1.43 \%$ to $4.27 \%$ vs $0-1$ men) and lack of condom use at last anal sex (OR 1.51, 95\% CI $1.07 \%$ to $2.12 \%$ vs condom use) were associated with detection of multiple anal HPV infections among MSM. Among MSW, $\geq 2$ female sex partners in the past 6 months (OR 1.81, 95\% CI $1.02 \%$ to $3.21 \%$ vs $0-1$ women) was associated with detection of multiple anal HPV infections while condom use at last vaginal sex was not associated with infection (no condom use: OR $0.90,95 \%$ CI $0.51 \%$ to $1.61 \%$ vs condom use)

Conclusions These data suggest that lowering the number of sex partners may reduce infection with multiple HPV types at the anal canal among MSM and MSW. Additionally, using condoms during anal sex among MSM, even among men with multiple partners, may reduce multiple anal HPV infections at the anal canal. Questions:alan.nyitray@moffitt.org

\section{1-S02.04 EVIDENCE OF HPV VACCINE EFFECTIVENESS IN REDUCING GENITAL WARTS: AN ANALYSIS OF CALIFORNIA PUBLIC FAMILY PLANNING ADMINISTRATIVE CLAIMS DATA, 2007-2009}

doi:10.1136/sextrans-2011-050109.10

H Bauer, G Wright, J Chow. Department of Public Health, Richmond, California, USA

Background The quadrivalent HPV vaccine, available in the US since 2006 , is recommended for females age 9-26. This vaccine prevents HPV types 6 and 11, which cause $90 \%$ of genital warts (GW). Because of the rapid development of GW after infection, monitoring GW trends may provide early evidence of population level vaccine effectiveness.

Methods Trends in GW diagnoses were assessed using clinical encounter claims data from the California Family Planning Access Care and Treatment program which serves low-income females and males. Following implementation of diagnostic coding requirements, reliable data on International Classification of Diseases 
(ICD-9) codes were available 2007 through 2009. GW cases were defined as unduplicated clients with either an ICD-9 diagnostic code for viral warts (078.10) or condyloma (078.11) or a National Drug Code (NDC) for pharmacy-dispensed imiquimod or podofilox. Inclusion of procedure codes for destruction of genital lesions was unnecessary because these claims closely correlated (>95\%) with appropriate ICD-9 codes. Denominators included unduplicated clients served. The proportions of clients with GW were stratified by age $(<21,21-25,26-30,31+)$, gender, and year. No data on vaccination status were available. Statistical significance of genderand age-specific trends was assessed using the Cochran-Armitage test for linear trend.

Results Between 2007 and 2009, an average of over 1735000 female and 248000 male clients were served annually. Total clients served increased each year for both females and males across all age groups, with the greatest increases seen among older age groups. Overall, $0.7 \%$ of females and $3.4 \%$ of males were diagnosed with GW. Between 2007 and 2009, GW diagnoses declined 19.4\% among females less than age 21 ( $p$ trend $<0.0001$ ), whereas GW diagnoses were stable or increasing among females in older age groups see Abstract O1-S02.04 table 1. No statistically significant declines in GW diagnoses were observed among males of any age group; however, significant increases were observed among older males.

Conclusions This analysis provides preliminary evidence that the HPV vaccine may be preventing GW among young women. Although using existing administrative claims data to assess trends in HPV-related diseases was inexpensive and expeditious, trends are ecological and may be explained by factors other than vaccination.

Abstract 01-S02.04 Table 1 Per cent change between 2007 and 2009 in GW diagnoses by gender and age group

\begin{tabular}{lccccc}
\hline \multirow{2}{*}{ Age group } & \multicolumn{2}{l}{ Females } & & \multicolumn{2}{l}{ Males } \\
\cline { 2 - 3 } & \% Change & $\mathbf{p}_{\text {trend }}$ & & \% Change & $\mathbf{p}_{\text {trend }}$ \\
\hline$<21$ & -19.4 & $<0.0001$ & & -3.3 & 0.328 \\
$21-25$ & -2.8 & 0.137 & & -4.1 & 0.061 \\
$26-30$ & +8.8 & 0.006 & & +12.6 & 0.0004 \\
$31+$ & +10.9 & 0.002 & & +11.4 & 0.004 \\
\hline
\end{tabular}

\section{1-S02.05 POPULATION BASED SURVEILLANCE FOR CERVICAL INTRAEPITHELIAL NEOPLASIA GRADE 3 AND ADENOCARCINOMA IN SITU IN THREE CENTRAL CANCER REGISTRIES, USA 2009}

doi:10.1136/sextrans-2011-050109.11

${ }^{1} \mathrm{E}$ W Flagg, ${ }^{2} \mathrm{~S}$ Deblina Datta, ${ }^{3} \mathrm{C}$ Lyu, ${ }^{3} \mathrm{~J}$ Nagaraja, ${ }^{4} \mathrm{G}$ Copeland, ${ }^{4} \mathrm{~W}$ Silva, ${ }^{5} \mathrm{E}$ Peters, ${ }^{5} \mathrm{~L}$ Cole, ${ }^{6} \mathrm{~T}$ Tucker, ${ }^{6} \mathrm{M}$ J Byrne, ${ }^{2} \mathrm{E} \mathrm{R}$ Unger, ${ }^{2} \mathrm{M}$ Saraiya, ${ }^{2} \mathrm{H}$ Weinstock. ${ }^{1}$ US Centers for Disease Control and Prevention, Atlanta, USA; ${ }^{2}$ Centers for Disease Control and Prevention, USA; ${ }^{3}$ Battelle Memorial Institute, USA; ${ }^{4}$ Michigan Cancer Registry, USA; ${ }^{5}$ Louisiana Tumour Registry, USA; ${ }^{6}$ Kentucky Tumour Registry, USA

Background Human papillomavirus (HPV) vaccine has been recommended routinely to 11-12-year-old US girls for cervical cancer prevention since 2006, and evaluation of the population impact of HPV vaccine is a critical need. In addition to measuring the impact of HPV vaccines on cervical cancer incidence, surveillance should include endpoints more proximal in time to HPV infection such as cervical intraepithelial neoplasia grade 3 (CIN3) and adenocarcinoma in situ (AIS). These immediate precursors to invasive cervical cancer manifest only $5-10$ years after HPV infection. Although CIN3/AIS are detected during cervical cancer screening, these lesions are not routinely reported to US central cancer registries (CCRs). Compared to other precursor lesions, CIN3/AIS show the most consistent inter-pathologist agreement in histopathology interpretation making them the most suitable precursor lesion surveillance endpoints.

Methods The Centers for Disease Control and Prevention conducted a project in three statewide CCRs to assess the feasibility of collecting data on CIN3/AIS lesions using existing registry infrastructure, a standardised case definition, and well-defined coding rules. State-specific vintage 2009 bridged-race postcensal population estimates were used to calculate incidence rates.

Results Statewide age-adjusted incidence rates of CIN3/AIS in 2009, using the 2000 US Standard Population, were 76.8 (Kentucky), 57.5 (Michigan), and 54.7 (Louisiana) per 100000 women. Highest rates were observed in those aged 20 to 29; rates among these women were 272.8 in Kentucky, 196.7 in Louisiana, and 192.6 in Michigan. Race was missing for $16 \%$ of records. Among records for which race was reported, incidence rates in Kentucky were highest for whites, while rates in Michigan were highest for blacks; in Louisiana rates did not differ significantly between whites and blacks. In each state, overall rates of CIN3/AIS were over sixfold higher than invasive cervical cancer rates. Only 3.8\% of cervical lesions were AIS.

Conclusions These results are the first reports of statewide population based incidence of CIN3/AIS in the US, and demonstrate that routine collection of CIN3/AIS lesions by cancer registries is feasible and could provide an earlier endpoint than cervical cancer with which to evaluate the impact of HPV vaccination in the US. Sentinel registries should be established to collect ongoing data on CIN3/AIS to monitor the impact of HPV vaccine in the US

\section{1-S02.06 DETECTION OF CERVICAL CANCER PRECURSORS AND ASSOCIATED HPV TYPES IN THE USA: HPV-IMPACT PRELIMINARY RESULTS}

doi:10.1136/sextrans-2011-050109.12

${ }^{1} \mathrm{~S}$ E Powell, ${ }^{1} \mathrm{~S}$ Hariri, ${ }^{1} \mathrm{M}$ Steinau, ${ }^{2} \mathrm{H}$ Bauer, ${ }^{3} \mathrm{~N}$ Bennett, ${ }^{4} \mathrm{~K}$ Bloch, ${ }^{5} \mathrm{~S}$ Schafer, ${ }^{6} \mathrm{~L}$ Niccolai, ${ }^{1} \mathrm{E}$ R Unger, ${ }^{1} \mathrm{~L}$ Markowitz. ${ }^{1}$ Centers for Disease Control and Prevention Atlanta, USA; ${ }^{2}$ Department of Public Health, California, USA; ${ }^{3}$ University of Rochester Medical Center, USA; ${ }^{4}$ Vanderbilt University Medical Center, USA; ${ }^{5}$ Oregon Health Authority, USA; ${ }^{6}$ Yale University, USA

Background Cervical intraepithelial neoplasia (CIN) grade 2 or 3 and adenocarcinoma in situ (AIS) (CIN2+) can be used to monitor HPV vaccine impact. This spectrum of preinvasive cervical lesions are commonly associated with multiple HPV types and detected through screening. This abstract describes baseline CIN2+ data and associated HPV types among defined populations of US females.

Methods As part of a vaccine impact monitoring project (HPVIMPACT), CIN2 + cases in females 18-39 years were reported from pathology laboratories in five catchment areas (CA, CT, NY, OR, TN). One diagnostic block was selected and unstained serial sections were prepared for PCR. Extracts from samples with residual lesion on both $\mathrm{H} \& \mathrm{Es}$ were used in Roche Linear array to detect and type HPV. CIN2/3 diagnosis rates were determined in catchment areas (CA, CT and NY) with complete case reporting. HPV typing data were analysed from all five defined catchment areas.

Results In 2008, rates per 1000 population in 18-39-year-old females were 2.8 in CA, 5.3 in CT and 4.9 in NY. In all five sites, CIN2 was most common (49\%), followed by CIN3 (31\%) and AIS $(2 \%)$. The proportion of lesions not distinguished by grade (CIN2/3) varied across sites (from 12 to $27 \%$ ). Median diagnosis age was 31 years in CA, 29 in CT, 27 in NY, 29 in OR, 28 in TN. Among 5035 18-39-year-old females, 1413 (28\%) specimens were tested; 96\% were HPV DNA positive. HPV16 was most prevalent (47\%), followed by HPV31 (11\%), HPV52 (9\%) and HPV51 (8\%). HPV18 prevalence was $5.4 \%$. HPV16 prevalence varied by diagnosis: $38 \%$ in CIN2, $51 \%$ in CIN2/3, and $59 \%$ in CIN3. 